\title{
Frontières
}

\section{Perspectives éthiques sur la signification de l'expression} Mourir dans la dignité

\section{Jocelyne Saint-Arnaud}

Volume 24, numéro 1-2, automne 2011, printemps 2012

L’aide médicale à mourir

URI : https://id.erudit.org/iderudit/1013080ar

DOI : https://doi.org/10.7202/1013080ar

Aller au sommaire du numéro

\section{Éditeur(s)}

Université du Québec à Montréal

ISSN

1916-0976 (numérique)

Découvrir la revue

Citer cet article

Saint-Arnaud, J. (2011). Perspectives éthiques sur la signification de l'expression : mourir dans la dignité. Frontières, 24(1-2), 11-17.

https://doi.org/10.7202/1013080ar
Résumé de l'article

Que signifie mourir dans la dignité ? Cet article aborde la question par des considérations de l'ordre d'une anthropologie philosophique. De ce point de vue, la «bonne mort» est celle qui est rapide et sans douleur. Ce que les gens craignent, c'est une mort prolongée, souffrante et vécue dans la dépendance. Selon les partisans de l'institutionnalisation des pratiques euthanasiques, mourir dans la dignité signifie pouvoir avoir accès à l'aide au suicide et à l'euthanasie en fin de vie ou en cas de maladies chroniques dégénératives ; leur argument majeur repose sur le respect de l'autonomie de la personne. La moralité de cette option est examinée à la lumière de quatre théories ou approches pertinentes en éthique de la santé. Ce sont le déontologisme, le situationnisme, l'utilitarisme et l'approche bioéthique. 


\section{Résumé}

Que signifie mourir dans la dignité? Cet article aborde la question par des considérations de l'ordre d'une anthropologie philosophique. De ce point de vue, la «bonne mort» est celle qui est rapide et sans douleur. Ce que les gens craignent, c'est une mort prolongée, souffrante et vécue dans la dépendance. Selon les partisans de l'institutionnalisation des pratiques euthanasiques, mourir dans la dignité signifie pouvoir avoir accès à l'aide au suicide et à l'euthanasie en fin de vie ou en cas de maladies chroniques dégénératives; leur argument majeur repose sur le respect de l'autonomie de la personne. La moralité de cette option est examinée à la lumière de quatre théories ou approches pertinentes en éthique de la santé. Ce sont le déontologisme, le situationnisme, I'utilitarisme et l'approche bioéthique.

Mots clés: mort - dignité -

déontologisme - situationnisme utilitarisme - bioéthique - approche par principes - euthanasie - suicide aide au suicide - éthique de la santé.

\section{Abstract}

What does "death with dignity" mean? First of all this paper addresses the question in terms of philosophical anthropology. From this perspective, the "good death" comes fast without suffering. A prolonged, painfull and others-dependant death is feared. Groups who promote institutionalization of euthanasia, consider that "death with dignity" ought to include access to euthanasia and suicide assistance at the end of life or in case of degenerative chronic diseases; their main argument is based on respect for autonomy. To discuss the morality of this option, four ethical theories or approaches relevant to ethics of health care have been chosen. These are deontologism, situationism, utilitarianism and bioethics.

Keywords: death - dignity deontologism - situational ethics utilitarianism - bioethics - principlism

- euthanasia - suicide - suicide assistance - health care ethics.

\section{Perspectives éthiques sur la signification de l'expression:}

Mourir dans la dignité

\section{Jocelyne Saint-Arnaud, Ph.D. (philosophie), professeure honoraire, Université de Montréal; chercheure associée au Centre de recherche en éthique de I'Université de Montréal (CRÉUM) et au Groupe inter-réseaux de recherche sur l'adaptation de la famille et de son environnement (GIRAFE).}

Qu'est-ce que la mort dans la dignité? Généralement, la mort dans la dignité est associée à une mort sereine et sans douleur. Cependant, selon les partisans d'une institutionnalisation des pratiques euthanasiques, avec ou sans décriminalisation et légalisation ${ }^{2}$, la mort dans la dignité devrait inclure un accès à l'euthanasie et à l'aide au suicide, pour des personnes en fin de vie ou des personnes atteintes de maladies chroniques dégénératives qui en ont fait la demande. Leur argumentation repose principalement sur le respect de l'autonomie de la personne. Cette option peut être évaluée à la lumière de certaines théories et approches en éthique de la santé, de manière à mettre en évidence le fondement de la dignité humaine et la place qu'elles accordent au respect de l'autonomie de la personne.

Dans un premier temps, nous examinons ce que signifie la mort dans la dignité dans une perspective d'anthropologie philosophique, puis dans la perspective des partisans de l'institutionnalisation des pratiques euthanasiques. Par la suite, la moralité de l'euthanasie est évaluée à partir de théories et approches éthiques qui ont des vues différentes, voire opposées sur la question.

\section{MOURIR DANS LA DIGNITÉ DU POINT DE VUE D'UNE ANTHROPOLOGIE PHILOSOPHIQUE}

Deux concepts sont en cause : la mort et la dignité. La mort, c'est la fin de l'humain sous ses aspects bio-psycho-sociaux. Tout un chacun sait ce qu'est la mort, depuis 
qu'il est jeune. Il a vu mourir des plantes ou des animaux, mais plus rarement des humains. La mort fait partie de la vie humaine, mais elle est tabou, on l'occulte, on la repousse.

Les techniques biomédicales ont modifié notre relation à la mort. Au début du siècle dernier, celui qui consultait un médecin n'était pas sûr d'y trouver un bénéfice. Il rencontrait par contre un professionnel qui faisait un examen physique et qui écoutait son histoire dans le but d'y trouver des indications diagnostiques. Aujourd'hui les tests ont remplacé, pour une bonne part, le travail diagnostique du médecin et la communication en prend pour son rhume, soumise aux impératifs de l'efficience... Mais la médecine a réalisé d'importantes victoires sur la mort. Pensons à tout ce qui peut être fait de nos jours pour prolonger la vie biologique et suppléer aux fonctions vitales défaillantes: réanimation, stimulateur cardiaque, intubation et ventilation, dialyse rénale, appareils de circulation extra-corporelle, transplantation d'organes, transfusion sanguine, bientôt thérapie génique, et tous les médicaments comme l'insuline, les anticoagulants, les bêtabloquants, etc. Jumelés à d'autres facteurs comme les progrès dans le domaine de l'hygiène et de la santé publique, les avancées médicales ont repoussé la mort. L'humain vit maintenant assez longtemp. pour connaître les affres de la démence. Le vieillissement est en effet le facteur de risque le plus important lié au développement de la démence. En 2008, 46\% des femmes de 85 ans et plus et $33 \%$ des hommes du même groupe d'âge en sont atteints (Société Alzheimer, 2010).

Ce que craignent les humains, ce n'est pas tant la mort que la douleur et la souffrance qui la précèdent. Des personnes interrogées dans le cadre d'une étude (Vig et Pearlman, 2004) ont décrit la bonne mort comme survenant durant le sommeil, rapidement, sans douleur ni souffrance. Elles craignent une mort lente et douloureuse, vécue dans la dépendance, en étant un fardeau pour les autres. Si le lecteur réfléchit à la mort qu'il souhaite, il y a tout lieu de croire que les mêmes constats ressortiront.

En quoi consiste la dignité? Question beaucoup plus difficile à répondre parce qu'il s'agit d'un concept plus abstrait. Y a-t-il une mort digne et une mort indigne? Tout dépend de la perception qu'on en a. Si plusieurs individus sont en train de mourir dans un séisme, trouvent-ils la mort digne ou indigne? Ce n'est sûrement pas des questions qu'ils se posent: ils souffrent et sont tout entiers à leur souffrance. La dignité ou l'indignité est dans le regard de l'Autre, de celui qui est en bonne santé, qui

juge et qui se dit: Que puis-je faire face à une telle réalité que la mort?

Idéalement, mourir dans la dignité signifie mourir paisiblement et confortablement, sans douleur physique, ni souffrance morale, et entouré des siens. C'est mourir sans tout l'attirail technique des soins intensifs et des soins d'urgence qui sont conçus pour sauver et prolonger des vies. Quand les traitements et les techniques deviennent un fardeau pour la personne en cause, les conditions de vie peuvent devenir indignes pour la personne qui porte son regard sur celle qui est diminuée physiquement et psychologiquement par la maladie au point de devenir totalement dépendante des techniques pour sa survie, celle qui est mourante qu'on s'obstine à réanimer, ou celle qui est irréversiblement comateuse à qui l'on donne des gavages pour la garder en vie. Ce regard induit en nous un questionnement sur le sens des interventions médicales. Pensons-nous au bien de la personne en cause ou refusons-nous, pour des raisons personnelles, professionnelles ou sociales, de considérer que la mort existe et qu'il y a des limites à la retarder? L'acharnement thérapeutique n'est pas étranger aux craintes entourant la mort et aux demandes d'euthanasie et d'aide au suicide.

Alors mourir dans la dignité, ce n'est pas mourir dans une urgence ou dans un

LA THÉORIE KANTIENNE DES DEVOIRS A ÉTÉ CRITIQUÉE PARCE QUE LES DEVOIRS PARFAITS GÉNĖRENT UNE OBLIGATION MORALE ABSOLUE (SANS EXCEPTION). QUE FAIRE QUAND DEUX DEVOIRS PARFAITS ENTRENT EN CONFLIT? KANT NE DONNE AUCUNE INDICATION À CE SUJET.

corridor à la vue de tous les passants, ni à l'unité des soins intensifs branchés de partout. C'est mourir chez soi ou dans une unité de soins palliatifs, où les techniques sont mises de côté au profit d'une approche humaniste du soin, qui tient compte de tous les aspects de la personne, y inclus ses sentiments et ses émotions. Suivant la pyramide de Maslow, les soins physiques sont prioritaires. On doit donc en premier lieu soulager la douleur. Une personne dont la douleur est insupportable pense à mourir; elle peut devenir suicidaire. De bons soins de fin de vie impliquent donc en premier lieu un soulagement adéquat de la douleur, ensuite il est possible de s'attaquer aux autres aspects de la souffrance: douleur psychologique et douleur morale.

\section{DIGNITÉ, EUTHANASIE ET AIDE AU SUICIDE}

Pour les partisans d'une légalisation de l'euthanasie, mourir dans la dignité signifie généralement que la personne en fin de vie ou celle qui est atteinte d'une maladie dégénérative puisse avoir accès à l'aide au suicide et à l'euthanasie ${ }^{3}$ quand elle en fait la demande et qu'elle éprouve des souffrances morales ou physiques intolérables $^{4}$. Certains d'entre eux, notamment les experts de la Commission royale du Canada, vont plus loin dans leur revendication et sont d'avis qu'une personne pour qui la vie n'a plus de sens, même si elle n'est pas en fin de vie, devrait avoir accès à ces pratiques ${ }^{5}$. Quelle que soit leur position, l'argument majeur des tenants des pratiques euthanasiques repose sur le respect de l'autonomie de la personne.

Pour évaluer la moralité des pratiques euthanasiques, examinons quatre théories et approches éthiques pertinentes au domaine de l'éthique de la santé et ayant des positions différentes, voire opposées. Les théories et approches retenues sont: le déontologisme, le situationnisme, l'utilitarisme et l'approche bioéthique. Elles ont été choisies pour leur définition du fondement de la dignité humaine et ce qui en découle du point de vue d'une éthique normative, et pour le rôle qu'ils accordent au respect de l'autonomie de la personne. 
respecter la vie, ne pas mentir et tenir ses promesses. Ces devoirs ne supportent aucune exception. Tout être humain, en tant qu'être rationnel est digne de respect, quelle que soit sa condition physique, que ses capacités intellectuelles soient actualisées ou non. L'être humain ne doit pas se suicider parce qu'il s'utiliserait comme simple moyen en vue d'une fin autre que lui-même. De la même façon, il doit respecter la vie d'autrui. L'euthanasie, le suicide et l'aide au suicide sont moralement inacceptables, quelles que soient les circonstances, parce qu'ils vont à l'encontre du devoir parfait du respect de la vie. Dans le cadre de la philosophie kantienne, la bienfaisance (ne pas laisser souffrir) constitue un devoir imparfait, c'est-à-dire un devoir que l'individu est libre d'accomplir quand et comment il le juge approprié. Les devoirs parfaits ont toujours priorité sur les devoirs imparfaits.

Cependant, si l'humain est maintenu en vie de manière disproportionnée, alors qu'il est mortellement et irréversiblement atteint, il devient un moyen pour les seuls fins de la technique. Prolongement de la machine, il est réifié et perd sa dignité d'être humain. Dans de telles conditions, l'arrêt des techniques de maintien des fonctions vitales devient une obligation morale, ce qui prohibe l'acharnement thérapeutique sans toutefois donner le feu vert aux pratiques euthanasiques.

La théorie kantienne des devoirs a été critiquée parce que les devoirs parfaits génèrent une obligation morale absolue (sans exception). Que faire quand deux devoirs parfaits entrent en conflit? Kant ne donne aucune indication à ce sujet. Et est-il toujours pertinent de donner priorité aux devoirs parfaits sur les devoirs imparfaits? D'autres théories ont des positions différentes sur la question.

\section{LE SITUATIONNISME}

Pour le situationnisme, ce sont les circonstances propres à chaque cas qui sont déterminantes dans le choix de l'action à entreprendre. Chacun interprète les valeurs propres à sa culture à la lumière de sa conscience. Selon Joseph Fletcher (1954), une personne pourrait juger que l'euthanasie est une solution moralement acceptable à titre exceptionnel, au nom du principe de l'amour d'autrui (agapê). C'est à cet auteur que l'on doit toutes les catégories (directe/indirecte, volontaire/ non volontaire, passive/active) qui ont défini l'euthanasie pendant de nombreuses années en Amérique du Nord et encore aujourd'hui dans certains pays d'Europe.

Son argument est le suivant: si les formes d'euthanasie indirecte (soulagement de la douleur) ${ }^{6}$ et passive (arrêt de traitement maintenant les fonctions vitales) sont moralement acceptables, pourquoi les formes directes et actives ne le seraient-elles pas? Pour Fletcher, si le bien le plus grand concerne l'intégrité et le bien-être personnels, alors l'euthanasie qu'elle soit directe ou indirecte, passive ou active, est moralement acceptable ${ }^{7}$. Ce sont ses fonctions supérieures qui confèrent à l'humain sa dignité. Elles valent plus que la vie biologique et les fonctions qui y sont rattachées. Cela justifie un contrôle de la mort au même titre qu'il existe un contrôle des naissances.

Pour le situationnisme fletcherien, l'aide au suicide et l'euthanasie sont des actes moralement acceptables exceptionnellement dans certaines circonstances.

\section{POUR LE SITUATIONNISME}

FLETCHERIEN, L'AIDE

AU SUICIDE ET L'EUTHANASIE

SONT DES ACTES

MORALEMENT ACCEPTABLES

EXCEPTIONNELLEMENT

DANS CERTAINES CIRCONSTANCES.

C'EST LA CONSCIENCE

INDIVIDUELLE QUI PEUT

EN JUGER.

C'est la conscience individuelle qui peut en juger. Une telle approche peut être critiquée à l'aide des théories déontologiques traditionnelles, notamment celle de Kant présentée plus tôt. On peut aussi arguer que ce qui est moralement acceptable à titre exceptionnel du point de vue d'une éthique individuelle n'est pas nécessairement acceptable du point de vue d'une éthique sociale.

\section{L'UTILITARISME}

Pour les tenants de l'utilitarisme, le suicide ou l'euthanasie ne sont pas des actes immoraux, si les conséquences positives qui en découlent sont supérieures aux conséquences négatives, pour l'ensemble des personnes qui en seraient affectées. Ce principe, appelé principe d'utilité, peut s'appliquer à l'action singulière (Mill, 1979) ou à la règle (Brandt, 1992). Ce qui importe pour la morale utilitariste, c'est d'agir en fonction du bien-être des personnes qui auront à subir les consé- quences de l'action en cause. En cela réside la dignité humaine.

Dans le cadre de l'utilitarisme appliqué à l'acte (actutilitarianism), on pourrait imaginer une situation où la personne est très malade et souffrante, et qu'elle demande l'euthanasie avec l'accord de sa famille; tout se passe bien, la famille a bien réagi, la personne décédée libère un lit et le personnel soignant est disponible pour donner des soins à une autre personne plus jeune qui pourra récupérer. Dans une telle situation, il semble n'y avoir que des conséquences positives et l'euthanasie est moralement acceptable. Cependant, imaginons que le médecin ne connaissant pas suffisamment la pratique euthanasique, ne donne pas la bonne dose de relaxant musculaire, que la mort ne survienne que trois à quatre heures suivant l'injection, que la famille est au chevet à se morfondre, que l'infirmière ne sait pas quoi faire, que le deuil devient très difficile pour cette famille. Alors il s'agirait pour ces personnes d'une mort indigne et les conséquences négatives étant supérieures aux conséquences positives pour toutes les personnes concernées, une telle euthanasie ne serait pas moralement acceptable selon l'utilitarisme appliqué à l'acte. Il est légitime de considérer que le contreexemple soulève un problème d'ordre technique qui pourrait facilement être éliminé. Si les médecins sont bien formés dans l'utilisation des produits à utiliser pour réussir une euthanasie, la mort par euthanasie pourrait avoir un impact positif sur les personnes directement impliquées.

Cependant, pour institutionnaliser des pratiques euthanasiques, il faudrait démontrer qu'il y aurait plus de bénéfices que de torts à en retirer pour l'ensemble de la population (Saint-Arnaud, 1995). Suivant l'utilitarisme appliqué à la règle (rule utilitarianism), il faut examiner les bénéfices et les inconvénients de la loi pénale actuelle concernant l'aide au suicide et l'euthanasie pour l'ensemble de la population, et les comparer aux bénéfices et aux inconvénients anticipés qui seraient générés par une nouvelle loi qui dépénaliserait les pratiques euthanasiques et éventuellement les légaliserait. Pour changer la loi, selon l'utilitarisme, il faudrait qu'une nouvelle loi qui permettrait l'aide au suicide et l'euthanasie apporte plus de bénéfices que de torts à l'ensemble de la population que la loi actuelle.

Il existe deux façons de vérifier si la loi pénale actuelle est préférable à une nouvelle loi du point de vue de l'utilitarisme appliqué à la règle. La première façon consiste à tenter de prévoir les impacts d'une telle loi sur les pratiques de fin de vie, en examinant les risques et les bénéfices anti- 
cipés pour les personnes concernées, par comparaison avec les avantages et les torts causés dans le cadre de la loi actuelle ${ }^{8}$.

De sérieuses réserves s'appliquent à une telle évaluation: 1) toutes les conséquences ne peuvent être prévues à l'avance; 2) les conséquences anticipées peuvent ne pas se présenter; 3) l'anticipation ne permet pas de prévoir l'importance des torts ou des bénéfices en termes d'incidence ou de gravité.

La deuxième façon consiste à examiner les résultats des études évaluatives ou autres, dans les pays où ces pratiques sont décriminalisées ou légalisées et à vérifier si les torts anticipés se vérifient ou non et si des torts non anticipés se manifestent. L'avantage de cette option réside dans le fait que l'incidence et la gravité des conséquences anticipées pourraient être vérifiées. Cette façon de faire permet aussi de vérifier s'il existe des dérives dans les pratiques. Ceux qui ont tenté de prévoir l'impact de ces pratiques sur les comportements des personnes en fin de vie et sur les professionnels qui s'en occupent sont souvent les opposants à toute décriminalisation ou légalisation de ces actes. Ils brandissent ce que les bioéthiciens ont appelé l'argument de la pente glissante, traduisant littéralement le terme slippery slope. Cet argument se présente de la manière suivante: d'abord on admet qu'une pratique est moralement acceptable pour un groupe ayant des caractéristiques définies et graduellement on est amené à étendre la pratique à des groupes vulnérables dont les caractéristiques ne correspondent pas à celles du groupe initial. De ce fait, nous sommes entraînés vers des pratiques qui sont moralement inacceptables selon les critères initiaux. Ce qui semblait éthiquement acceptable au départ ouvre la porte à des pratiques éthiquement inacceptables.

On peut trouver un exemple de telles dérives dans la loi néerlandaise de 2001 par rapport aux balises émises en 1984. Selon les lignes directrices de 1984, seules les personnes aptes à participer aux décisions peuvent demander l'euthanasie (Horgan, 1991). Mais la Loi des pompes funèbres d'avril 2001, autorise le médecin à accepter des demandes d'euthanasie de la part de mineurs avec l'assentiment ou le consentement des parents ${ }^{9}$ et de la part de personnes devenues inaptes à la condition qu'elles l'aient demandée dans un testament de vie (Griffiths, Weyers et Adams, 2008). De plus, depuis 2005, le protocole de Groningen autorise l'euthanasie des nouveaux-nés et des bébés lourdement handicapés. Paradoxalement, ce sont les principes d'égalité devant la loi et d'équité qui justifient un tel glissement.
CE QUI IMPORTE

POUR LA MORALE UTILITARISTE,

C'EST D'AGIR EN FONCTION

DU BIEN-ÊTRE DES PERSONNES

QUI AURONT À SUBIR

LES CONSÉQUENCES DE L'ACTION

EN CAUSE. EN CELA

\section{RÉSIDE LA DIGNITÉ HUMAINE.}

L'utilitarisme appliqué à la règle est moins vulnérable aux critiques que l'utilitarisme appliqué à l'acte. Mais il faut être prudent, car les facteurs contextuels varient d'un pays à l'autre et influent sur les conséquences réelles de l'introduction d'une nouvelle loi. À titre d'exemple, citons le cas de la Belgique qui, en légalisant l'euthanasie, a aussi obligé les hôpitaux à consacrer un pourcentage de leurs lits à des soins palliatifs, alors que les Pays-Bas ont décriminalisé des pratiques euthanasiques à une époque où les soins palliatifs commençaient à peine à se développer.

\section{L'APPROCHE BIOÉTHIQUE}

La bioéthique américaine avec des auteurs comme Veatch et Englehardt a fait la promotion du respect des volontés de la personne et du consentement libre et éclairé dans le domaine des soins de santé. Veatch (1972) présente la relation médecin-patient sur la base d'une entente contractuelle en fonction de laquelle les valeurs du patient servent de cadre de référence à la décision médicale. De ce point de vue, le patient garde un contrôle sur sa vie et sa santé quand des décisions de soins importantes sont en cause. Pour les soins usuels, le médecin bénéficie de la confiance de son patient à moins que les termes du contrat ne soient pas respectés et que le contrat soit rompu. L'euthanasie et l'aide au suicide concernent de graves décisions; elles peuvent faire l'objet d'une entente entre le médecin et son patient. À ce niveau décisionnel, ce sont les valeurs du patient qui priment. Si une telle décision heurte les valeurs morales du médecin, il a la liberté de refuser une telle entente. Au niveau sociétal, c'est la communauté concernée qui joue le rôle de contractant et qui opère un contrôle sur les décisions générales de santé, alors que les médecins sont responsables des décisions médicales au jour le jour. Si donc la société concernée souhaite une institutionnalisation des pratiques euthanasiques, elle devrait être mise en place. Dans cette approche, le lieu de la décision relève du patient sur le plan individuel et de la communauté civile sur le plan sociétal. Les décisions prises ne sont pas de l'ordre du consensus et les droits des patients sont supérieurs à ceux des médecins qui, en fait, se résument au droit de retrait.

Quant à Englehardt (1986), il est d'avis que, dans une société pluraliste, on ne peut se référer à une échelle de valeurs canonique pour juger de l'action à entreprendre. La liberté individuelle y tient donc une grande place. Dans un article sur le suicide, Lebacqz et Englehardt (1980) favorisent le respect de l'autonomie de la personne au nom de la liberté individuelle qui est à la base de l'entente contractuelle qui lie les êtres humains entre eux. Dans une société pluraliste, selon ces auteurs, le seul postulat éthique reconnu concerne le respect mutuel, ce qui implique de respecter ses engagements et de ne pas utiliser les autres sans leur consentement. En cela réside la dignité humaine. L'être humain a un droit à la mort, mais il doit respecter les engagements qu'il a pris envers les autres; une personne qui n'a pas d'engagements envers d'autres personnes peut moralement se donner la mort ou autoriser une autre personne à le faire (Englehardt, 1986). De ce point de vue, le principe du respect de l'autonomie est limité par celui de responsabilité.

Dans l'approche par principes de Beauchamp et Childress (2009), le respect de l'autonomie de la personne est certes un devoir, mais l'obligation qu'il crée n'est pas absolue. Elle est limitée par les autres principes: bienfaisance, non-malfaisance et justice. Ces principes ne sont pas hiérarchisés entre eux. Ils sont conçus comme universels et proviennent d'origines empiriques diverses et du sens commun. Ils génèrent des devoirs qui doivent être respectés par les professionnels de la santé qui veulent agir de manière éthique. En agissant de manière à respecter les quatre principes bioéthiques, l'individu agit avec dignité et traite l'autre avec dignité dans les décisions de soins que la fin de la vie soit en cause ou non.

Les devoirs suscités par les principes sont de nature prima facie, c'est-à-dire que s'ils entrent en conflit, la spécification de leurs exigences au regard des faits pertinents permet soit d'en effectuer un équilibrage, soit de donner priorité à l'un ou l'autre des principes en conflit. Il n'y a donc pas d'ordre de priorité préétabli entre les principes, ce que l'on trouve chez Kant entre les devoirs parfaits et les devoirs imparfaits ou chez les utilitaristes pour qui le principe d'utilité est prioritaire. C'est l'interprétation des principes 
qui sera effectuée en fonction de chacune des situations concrètes et singulières qui déterminera un ordre de priorité en cas de conflits non résolus, rendant ainsi possible la prise de décision.

L'approche par principes peut être utilisée au niveau micro-éthique, c'est-à-dire dans la résolution des problèmes éthiques liés aux demandes d'euthanasie et d'aide au suicide effectuées en pratique clinique. Selon nous, cette approche peut aussi être utilisée au niveau macro-éthique, c'està-dire dans la résolution des problèmes éthiques suscités par les demandes d'institutionnalisation, de décriminalisation et de légalisation des pratiques euthanasiques, ce qui relève de la santé publique.

Selon l'approche par principes telle qu'appliquée à la demande d'euthanasie en éthique clinique, il est clair que

1. la personne malade devrait en avoir fait la demande en ayant en main toutes les informations pertinentes à sa décision et sans subir de pressions indues, en accord avec la doctrine du consentement libre et éclairé (respect de l'autonomie);

2. les douleurs ou les souffrances en cause ne devraient pas pouvoir être soulagées autrement que par une pratique euthanasique, la vie devenant un trop lourd fardeau (bienfaisance et nonmalfaisance);

3. d'autres personnes placées dans les mêmes circonstances et répondant aux mêmes conditions devraient y avoir accès (équité).

Aux exigences de l'approche par principes de Beauchamp et Childress, nous ajoutons que

4. la décision devrait provenir d'une démarche qui tienne compte du tout de la personne, en partenariat avec elle, dans une démarche globale de soin et empreinte d'humanisme (caring $)^{10}$.

Si on applique les principes précédents au domaine de la santé publique ${ }^{11}$ duquel relève la demande d'institutionnalisation des pratiques euthanasiques dans les établissements de santé, les conditions suivantes devraient être respectées:

1. il devrait y avoir une demande de la population à cet effet; cette demande devrait être faite en connaissance de cause, incluant des connaissances sur ce que sont les pratiques euthanasiques et un état de la situation actuelle; enfin, la demande devrait être faite sans pressions de la part de groupes dont les intérêts et l'idéologie vont à l'encontre $\mathrm{du}$ bien commun (respect de l'autonomie populationnelle et décision libre et éclairée) ;

2. les bénéfices prévus pour l'ensemble de la population dans le fait d'institutionnaliser ces pratiques devraient être

DANS CETTE APPROCHE, LE LIEU DE LA DÉCISION RELÈVE

DU PATIENT SUR LE PLAN INDIVIDUEL ET DE LA COMMUNAUTÉ

CIVILE SUR LE PLAN SOCIÉTAL. LES DÉCISIONS PRISES

NE SONT PAS DE L'ORDRE DU CONSENSUS ET LES DROITS DES PATIENTS

SONT SUPÉRIEURS À CEUX DES MÉDECINS QUI, EN FAIT,

SE RÉSUMENT AU DROIT DE RETRAIT.

supérieurs aux risques à encourir et le rapport bénéfices-risques associé à la nouvelle loi devrait être supérieur à celui engendré par la pratique actuelle (supériorité des bénéfices sur les risques); de plus, des mesures doivent être prises pour minimiser les risques, advenant le cas où les nouvelles pratiques sont institutionnalisées.

3. l'accès aux pratiques euthanasiques selon les mêmes conditions et sans discrimination devrait être garanti (équité) ;

4. et, enfin, les milieux de pratique devraient offrir des soins de qualité en fin de vie selon une approche globale de soins, incluant une expertise en soulagement adéquat de la douleur, autant que des connaissances adéquates en matière de pratiques euthanasiques (caring).

Selon l'approche par principes, les conditions énumérées plus haut devraient toutes être remplies avant de répondre positivement à une demande d'euthanasie en clinique et avant de donner libre cours à une institutionnalisation des pratiques, que la loi soit modifiée ou non. En clinique, il relève de la conscience individuelle des médecins de répondre positivement à une demande euthanasique, sachant que de telles pratiques vont à l'encontre de certaines règles déontologiques et de la loi actuelle. En santé publique, il faudrait s'assurer par référendum que la population se prononce sur la question, après avoir été clairement informée des éléments essentiels à une prise de décision éclairée (nature des actes en cause, état de la situation, bénéfices et torts anticipés). Il faudrait aussi s'assurer de connaître la situation actuelle à l'égard des pratiques d'euthanasie au moyen d'études scientifiquement fondées et apportant des données de nature empirique fiables, de manière à éviter de légiférer sur la base de cas exceptionnels $^{12}$. Enfin, il serait éthiquement inacceptable que la piètre qualité des services, le manque d'accès à des soins palliatifs, l'acharnement thérapeutique, les coûts des traitements et des médicaments, l'absence de formation en soulagement de la douleur, soient des incitatifs à demander l'euthanasie ou à institutionnaliser ces pratiques. Ce dernier point impose une responsabilité morale aux différents paliers de gouvernement impliqués dans l'administration des soins de santé.

Nous avons donné des indications générales sur la manière d'effectuer l'application des principes, il reste à faire l'analyse des exigences de ces principes en fonction des contextes québécois et canadien. Une telle analyse ne peut être effectuée dans le cadre restreint de cet article; elle demeure toutefois essentielle pour juger de la pertinence de changer la loi canadienne ou même d'institutionnaliser des pratiques euthanasiques en accord avec le Barreau, l'Association des établissements de santé et de services sociaux et le Collège des médecins du Québec, en tant qu'exceptions à la loi canadienne.

\section{SYNTHÈSE DE L'ANALYSE}

Les théories ou approches présentées peuvent être critiquées les unes par les autres. Certaines sont incompatibles; c'est le cas de la philosophie kantienne par rapport aux autres théories examinées. Les philosophies kantienne et fletcherienne fondent la dignité humaine sur le caractère rationnel de l'être humain, mais tiennent des positions contraires quant à la moralité des pratiques euthanasiques. L'utilitarisme fonde la moralité de l'action sur ses conséquences bonnes ou mauvaises, ce qui fait qu'une euthanasie ou une aide au suicide peut être moralement bonne ou mauvaise, selon que les personnes qui en sont affectées en retirent plus de bénéfices que de torts ou inversement. Pour institutionnaliser des pratiques euthanasiques, il faudrait démontrer que les nouvelles règles apporteraient plus de bénéfices que de torts à l'ensemble de la population. Il ne s'agit donc pas uniquement d'éviter les poursuites judiciaires aux médecins qui acceptent de pratiquer un acte euthanasique, mais encore de montrer que les groupes vulnérables ne seraient pas plus à risque d'être euthanasiés dans un tel contexte ${ }^{13}$. L'approche contractuelle de Veatch ne clarifie pas les liens entre 
l'action individuelle et les valeurs sociales faisant l'objet d'un contrat. Elle pourrait donner à penser que la décision euthanasique relève d'une entente privée entre le médecin et son patient et que la loi n'a pas à s'en mêler, si socialement la pratique est acceptable ${ }^{14}$. L'approche contractuelle définie par Englehardt limite la liberté d'avoir recours aux pratiques euthanasiques par la responsabilité de remplir ses engagements; cependant, si des personnes majeures et aptes libéraient le contractant des engagements pris envers elles et qu'il n'y avait pas de personnes inaptes ou d'enfants en cause, la personne serait libre de mettre fin à sa vie en ayant recours à l'euthanasie ou à l'aide au suicide. Par ailleurs, les principes éthiques sont plus exigeants car ils imposent des devoirs à être remplis, faute de quoi les pratiques euthanasiques seraient inacceptables. Par ailleurs, si ces conditions étaient remplies, les principes pourraient être garants de conditions moralement acceptables pour les pratiques d'euthanasie et d'aide au suicide. Cependant, les analyses contextuelles auxquelles l'application des principes doit donner lieu n'ont pas encore été effectuées de manière systématique, on ne peut donc pas présumer à l'avance que l'institutionnalisation des pratiques euthanasiques serait éthiquement acceptable dans le contexte québécois et canadien des soins de santé.

Ce que la présente étude met en évidence, c'est que certaines théories éthiques approuvent des pratiques euthanasiques qui respectent certaines conditions. Il est difficile cependant de justifier un changement dans les pratiques institutionnelles ou dans la loi sur l'unique base de la conscience individuelle, tel que le propose le situationnisme de Fletcher, sans considérer les conséquences anticipées d'un tel changement, ce qui relève de l'utilitarisme appliqué à la règle. Plus encore, à l'évaluation des bénéfices par rapport aux torts anticipés pour l'ensemble de la population que l'approche par principes intègre dans l'application des principes de bienfaisance et de non-malfaisance dans le domaine de la santé publique s'ajoutent des obligations à respecter provenant du respect de l'autonomie, de l'équité et du caring. En plus d'être plus complète, cette dernière approche a l'avantage de mettre l'accent sur des écueils à éviter avant d'effectuer de tels changements.

S'il est juste que les gens craignent une mort lente et prolongée encouragée en milieu hospitalier par tous les moyens dont la médecine dispose pour prolonger la vie de personnes en fin de vie ou dont les capacités vitales sont minimales, les théories éthiques choisies n'approuveraient certainement pas l'acharnement thérapeutique. Certaines d'entre elles approuveraient des pratiques euthanasiques selon certaines conditions. Encore faudrait-il démontrer que non seulement les bénéfices envisagés seraient supérieurs aux torts à encourir et à ce sujet, l'utilitarisme et l'approche par principes se rejoignent, mais encore que la population et les groupes qui demandent l'institutionnalisation de ces pratiques le font en toute connaissance de cause. À ce sujet, des analyses plus poussées sont à compléter.

\section{Bibliographie}

BATTIN, M.P., VAN DER HEIDE, A., GANZINI, L., VAN DER WAL, G. et ONWUTEAKA-PHILIPSEN, B.D. (2007). "Legal physician-assisted dying in Oregon and the Netherlands: Evidence concerning the impact on patients in "vulnerable" groups », Journal of Medical Ethics, vol. 33, $\mathrm{n}^{\mathrm{o}} 10$, p. 591-597.

BEAUCHAMP, T.L. et J.F. CHILDRESS (2009 [1979]). Principles of Biomedical Ethics, $5^{\mathrm{e}}$ éd., Oxford, Oxford University Press.

BRANDT, R.B. (1992). Morality, Utilitarianism, and Rights, Cambridge, Cambridge University Press.

CONSEIL D'EUROPE (s.d.). Études de législation, pour les Lois néerlandaises, en ligne, <http://www.senat.fr/lc/lc109/ lc1092.html>, consulté le 2010-02-15.

ENGLEHARDT, H. T. Jr. (1986 A). The Foundation of Bioethics, Oxford, Oxford University Press.

FLETCHER, J. (1954). "Euthanasias », dans Morals and Medicine, Princeton, Princeton University Press, p. 203-204.

FLETCHER, J. (1966). Situation Ethics: The New Morality. Philadelphie, The Westminster Press.

FLETCHER, J. (1967). Moral Responsibility: Situation Ethics at Work, Philadelphie, Westminster Press.

GRIFFTHS, J., H. WEYERS et M. ADAMS (2008). Euthanasia and Law in Europe, Oxford \& Portland (OR), Hart Publishing.

HORGAN, J. (1991). «Death with dignity: The Dutch explore the limits of a patient's right to die», Scientific American, mai, p. $17-20$.

KANT, E. (1988 [1785]). Fondements de la métaphysique des mours, trad. de J. Muglioni, Paris, Bordas.

LEBACQZ, A.K. et H.T. ENGLEHARDT Jr. (1980). "Suicide and the patient's right to reject medical treatment», dans Death, Dying, and Euthanasia, D.J. Horan et D. Mall (dir.), Frederick (MD), Alethia Books, p. 669-705.

LE BRETON, D. (2000). «Aspects sociaux et culturels de la douleur ", dans Soins infirmiers et douleur: évaluation de la dou- leur, modalités du traitement, psychologie du patient, interventions infirmières, C. Metzger, A. Muller, M. Schwetta et C. Walter (dir.), Paris, Masson, 2000, p. 120-127.

MILL, J.S. (1979 [1863]). Utilitarianism, Indianapolis, Bobbs-Merrill.

RACHELS, J. (1989). "Active and passive euthanasia ", dans Euthanasia: The Moral Issues, R.B. Baird et S.E. Rosenbaum (dir.), New York, Prometheus Books, p. 45-51.

SAINT-ARNAUD, J. (1995). "Enjeux éthiques soulevés par la décriminalisation de l'aide au suicide et de l'euthanasie", Frontières, vol. 8, n 1, p. 24-27.

SAINT-ARNAUD, J. (2009). L'éthique de la santé: guide pour l'intégration de l'éthique dans les pratiques infirmières, Montréal, Gaëtan Morin/Chenelière Éducation.

SOCIÉTÉ ALZHEIMER (2010). Raz de marée: Impact de la maladie d'Alzheimer et des affections connexes, 2010, en ligne, <http://www.alzheimer.ca/docs/RisingTide/Secured_As \%20Ring \%20Rising $\% 20$ Tide \%20Full \%20Report $\% 20$ Final $\% 20$ FR pdf $>$, consulté le 2011-03-14.

VEATCH, R.M. (1972). «Models for ethical medicine in a revolutionary age ", Hastings Center Report, vol. 2, n 3, p. 5-7.

VIG, E. et R.A. PEARLMAN (2004). «Good and bad dying from the perspective of terminally ill men ", Archives of Internal Medicine, vol. 164, n 9, p. 997-981.

\section{Notes}

1. Une version de ce texte a fait l'objet d'une conférence dans le cadre des Journées Philopolis, UQAM, le 19 mars 2011.

2. Une décriminalisation implique de retirer les articles du Code criminel qui condamnent les pratiques d'euthanasie et d'aide au suicide, une légalisation implique d'encadrer ces pratiques dans une loi et une institutionnalisation implique de mettre ces pratiques en place dans nos institutions et donc de les encadrer par des règles institutionnelles, sans nécessairement changer la loi.

3. L'aide au suicide implique que la personne directement concernée se donne elle-même la mort avec l'aide d'une autre personne, en l'occurrence un médecin qui fournit l'ordonnance qui permet à la personne suicidaire de s'enlever la vie. L'euthanasie est effectuée par une autre personne que la personne en cause, en l'occurrence un médecin ou une infirmière qui fait une injection létale, habituellement un barbiturique et un relaxant musculaire.

4. Les pays ou les États qui ont légalisé l'euthanasie ou l'aide au suicide ont spécifié des balises impliquant une demande de la part du patient et la présence de souffrances physiques ou mentales intolérables.

5. Société royale du Canada, Rapport sur la prise de décision en fin de vie. Novembre 2011. $<$ http://www.rsc.ca/documents/RSCEndofLifeReport2011_FR_Formated_FIANL.pdf $>$ (rapport complet)

6. Le soulagement de la douleur en accord avec les pratiques médicales admises n'entraîne pas la mort, au contraire il prolonge la vie. 
7. Le philosophe James Rachel (1989), utilisant l'exemple du bébé qui se noie dans son bain sans être secouru et le bébé que l'on noie en lui maintenant la tête sous l'eau, en vient à la même conclusion. Il est juste de dire que ce n'est pas le fait d'agir ou de ne pas agir qui caractérise ce qui est moral ou pas. Dans l'exemple de Rachel, les deux attitudes sont moralement inacceptables. De plus, s'il s'agit de comparer l'injection létale à l'arrêt de traitement, on ne peut pas dire qu'arrêter un traitement, c'est ne rien faire par la suite. $\mathrm{Au}$ contraire, on procure alors des soins de confort.

8. Richard Brandt, au chapitre 18 de son livre Morality, Utilitarianism, and Rights (1992), compare les bénéfices et les torts des pratiques actuelles d'avortements aux États-Unis avec les bénéfices et les torts d'une nouvelle loi qui permettraient l'euthanasie des bébés lourdement handicapés après la naissance. Il en conclut qu'il serait plus moral d'éliminer les bébés lourdement handicapés à la naissance, notamment parce que, dans les pratiques actuelles, des bébés sains sont avortés à la suite de faux diagnostics prénataux.

9. L'assentiment des parents est nécessaire pour les mineurs de 16 à 18 ans et le consentement des parents est nécessaire pour les mineurs de 12 à 16 ans.

10. Dans cette approche par principes, un principe de caring a été intégré. Pour en savoir plus, lire Saint-Arnaud (2009).

11. Saint-Arnaud (2009) a développé une approche par principes appliquée à la santé publique.

12. L'euthanasie était pratiquée aux Pays-Bas quand l'Association royale médicale hollandaise, la Commission d'État sur l'euthanasie et le gouvernement hollandais ont défini des balises pour encadrer la pratique en 1984 . Aujourd'hui, on ne sait pas quelle est l'incidence des pratiques euthanasiques au Québec.
13. Un article de Battin et ses collègues (2007) a comparé l'Oregon et les Pays-Bas pour examiner le risque encouru par les groupes vulnérables à recevoir l'euthanasie et l'aide au suicide. Cet article n'est pas crédible pour plusieurs raisons. D'abord, il a été accepté pour publication le jour de sa réception, le 10 juillet 2007(tel qu'indiqué p. 591), ce qui laisse croire qu'il n'a pas été évalué par les pairs. Deuxièmement, l'étude compare les Pays-Bas à l'Oregon, alors qu'en Oregon seul le suicide médicalement assisté est légalisé et aux Pays-Bas, le taux de suicide médicalement assisté est très bas, les personnes désirant abréger leur vie ont plutôt recours à l'euthanasie. Concernant les Pays-Bas, l'article combine des résultats d'études gouvernementales à d'autres études qui n'ont pas les mêmes méthodes de recherche. Les auteurs reconnaissent que « les différences méthodologiques des études originales rendent impossible de déterminer avec certitude l'incidence réelle de la mort assistée chez plusieurs des groupes vulnérables étudiés» (p. 594). Il y a aussi des incohérences dans l'interprétation des statistiques gouvernementales de 2005. Ainsi, concernant les femmes, on lit que les taux de recours à la mort assistée sont légèrement plus élevés que celui des hommes, alors que le tableau 1 montre que le pourcentage d'hommes aidés à mourir $(n=1350$ ou $56 \%)$ est beaucoup plus élevé que chez les femmes $(n=1050$ ou $44 \%)$ (p. 595). Dans ce groupe, comme dans les autres groupes vulnérables, il n'y a aucune mention de calculs effectués par des tests statistiques paramétriques, nous n'avons donc jamais d'indication montrant que des différences entre les groupes pourraient être statistiquement significatives. Sur la race et l'ethnicité, les auteurs indiquent qu'il n'y a pas de données gouvernementales à ce sujet, mais concluent quand même qu'il n'y a pas de risque plus grand d'obtenir de l'aide à mourir pour ce groupe, sur la base que «même les plus volubiles opposants à la mort assistée n'ont pas manifesté que l'euthanasie ou le suicide assisté s'était imposé plus fréquemment aux groupes raciaux stigmatisés ou au minorités ethniques» (trad. libre, p. 594).

14. Cette position est celle de médecins néerlandais qui ne rapportent pas les actes euthanasiques au coroner, comme la loi le stipule. 